\title{
Endoscopic ultrasonographic features of gastric mucosa-associated lymphoid tissue lymphoma with a 'pseudocircumvolutory' appearance
}

\author{
João Santos-Antunes $M D^{1,2}$, Susana Lopes $M D^{1}$, Manuel Sobrinho-Simões $M D^{3}$,
}

Hélder Cardoso MD ${ }^{1}$, Guilherme Macedo MD PhD ${ }^{1}$

\section{CASE PRESENTATION}

A 45-year-old man with a history of drug abuse, alcoholism and heavy smoking habits was referred to the hepatology outpatient clinic because of the presence of anti-hepatis $B$ virus (HBV) surface antigen, anti-HBV core antigen and anti-hepatitis $\mathrm{C}$ virus antibodies. He was being treated with tiapride and methadone.

He had no anemia, a normal white blood cell count, negative autoimmune serology, undetectable HBV DNA and hepatitis $\mathrm{C}$ virus RNA, and mildly elevated aminotransferase levels.

Immunoglobulin (Ig) M and IgA levels were elevated, with a monoclonal peak in protein electrophoresis. Myelography and bone biopsy

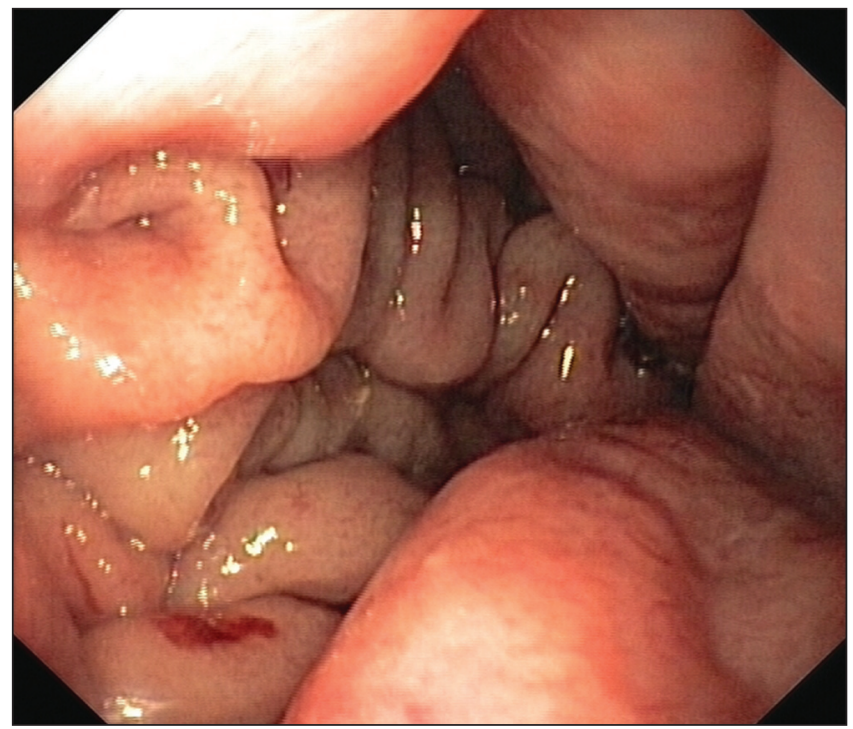

Figure1) Endoscopic image of thick gastric folds

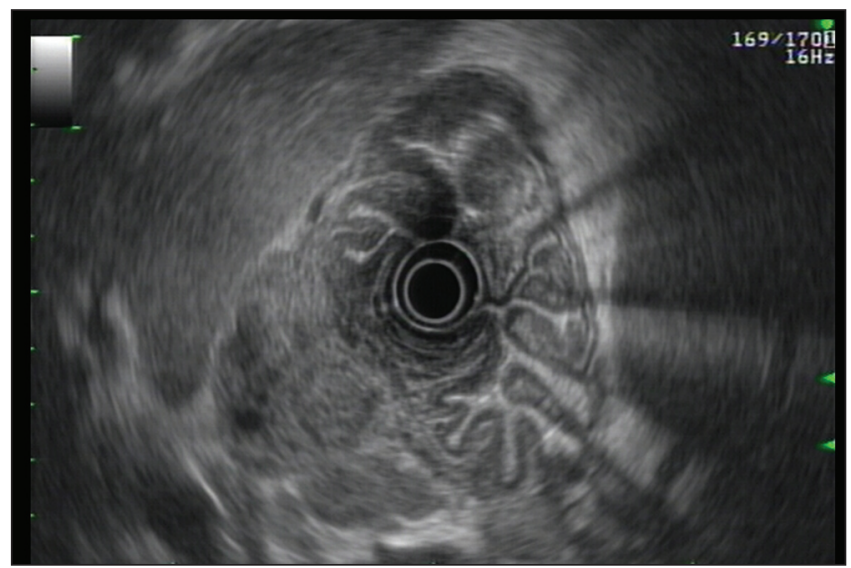

Figure 2) Endoscopic ultrasound image of the 'pseudocircumvolutory' appearance of the stomach revealed a cytological phenotype of Waldestrom macroglobulinemia with bone marrow involvement of small B cell lymphocytes, compatible with lymphoplasmacytoid lymphoma. An abdominal ultrasound and computed tomography scan revealed a thick gastric wall, and retroperitoneal and mesenteric adenopathies, raising suspicion for malignancy.

Esophagogastroduodenoscopy was remarkable for diffuse hard and edematous gastric folds of the body and fundus with some erosions (Figure 1). Endoscopic ultrasonography showed an asymmetrically thickened gastric wall ( $20 \mathrm{~mm}$ maximum), with disease limited to the mucosa and preservation of wall layers, and adenopathies (Figures 2 to 4). Biopsies were performed and histology revealed dense infiltration of mucosa by small B cells with irregular nuclei, plasmacytic differentiation and formation of lymphoepithelial lesions, strongly suggestive of mucosa-associated lymphoid tissue (MALT) lymphoma.

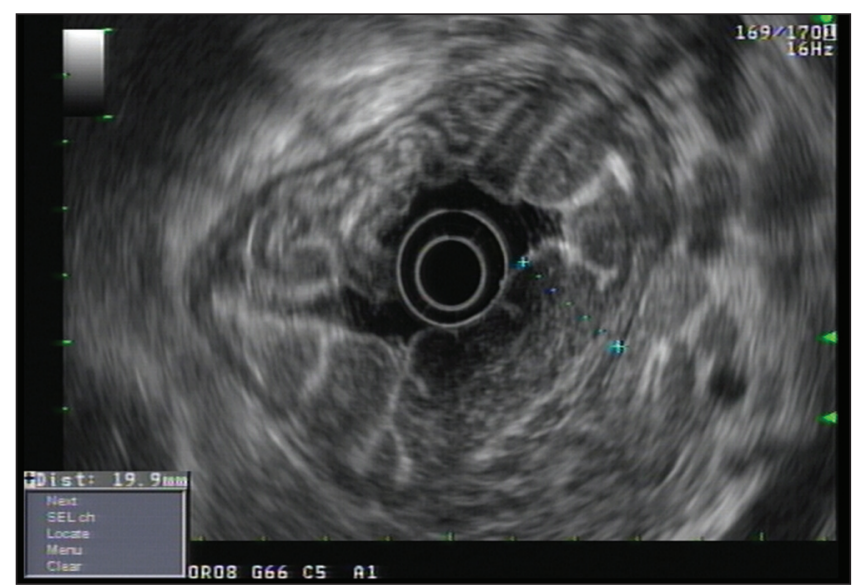

Figure 3) Endoscopic ultrasound image showing asymmetric involvement of the gastric wall

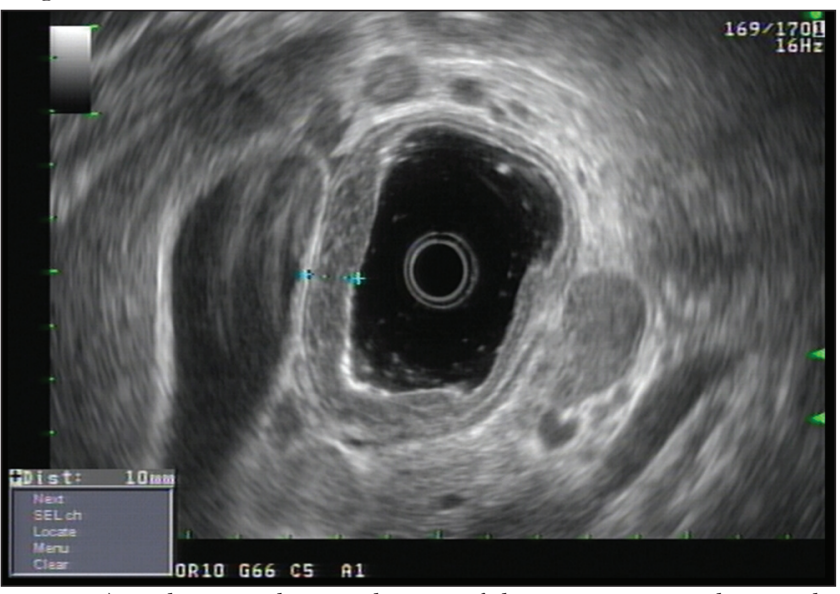

Figure 4) Endoscopic ultrasound image of the gastric antrum showing the preservation of the wall lavers

\footnotetext{
${ }^{1}$ Department of Gastroenterology, Faculty of Medicine, Centro Hospitalar S. João; ${ }^{2}$ Department of Biochemistry, Faculty of Medicine, University of Porto;

${ }^{3}$ Deparment of Hematology, Centro Hospitalar S. João, Porto, Portugal

Correspondence: Dr João Santos-Antunes, Gastroenterology Department, Centro Hospitalar S. João. Al. Prof. Hernâni Monteiro $4200-319$ Porto,

Portugal. Telephone 351-22-551-2100,fax 351-22-502-5766, e-mail joao.claudio.antunes@gmail.com

Received for publication July 11, 2013. Accepted September 15, 2013
} 
The patient is currently being treated for gastric MALT lymphoma with bone marrow involvement.

\section{DISCUSSION}

Gastric lymphomas are responsible for $5 \%$ of gastric neoplasms, with local extranodal lymphomas occurring most frequently. MALT-type lymphomas account for $8 \%$ of all non-Hodgkin lymphomas and for $40 \%$ of gastric lymphomas. Waldestrom macroglobulinemia is rarely associated with this type of lymphoma, particularly when it is located in the stomach.

MALT lymphoma can present as a single (mainly in the gastric antrum) or multiple gastric lesions. Endoscopy can reveal infiltrative and ulcerated masses, or simply congestive thick gastric folds with mucosal erosions. The most typical histological presentation is the presence of lymphoepithelial lesions (with invasion of glands and crypts by tumoural cells) and lymphocytes with irregular nuclei, as in the case described. Endoscopic ultrasound can reveal neoplastic involvement of deeper layers or, as in the case described, disease limited to the mucosa, with all other gastric layers well identified and delimitated.

Approximately $75 \%$ of patients with gastric MALT lymphoma present with disease limited to the stomach (stage I) at diagnosis, with a survival rate of $>80 \%$ at five years. In our case, the patient already presented with medullar involvement (stage IV); therefore, a therapeutic regimen used in advanced non-Hodgkin lymphomas was prescribed. We expect an indolent disease progression, with transitory response to chemotherapy. 


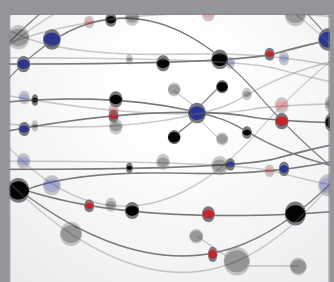

The Scientific World Journal
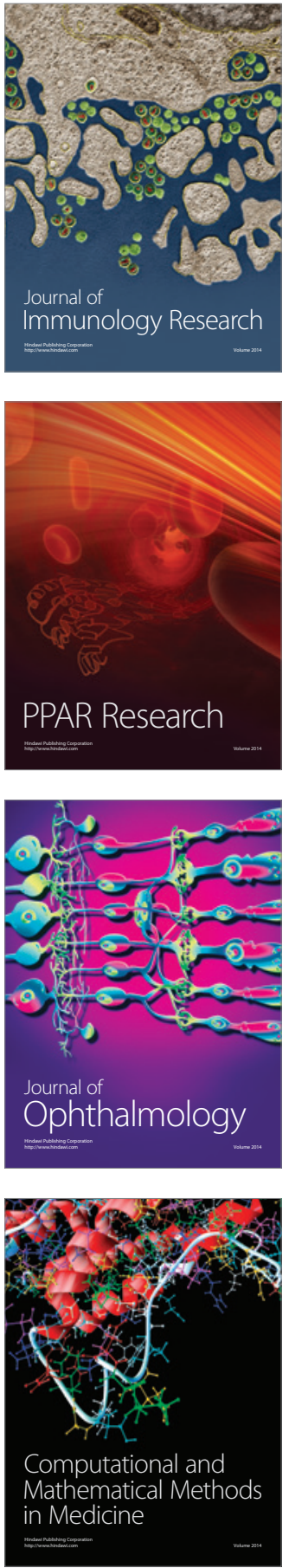

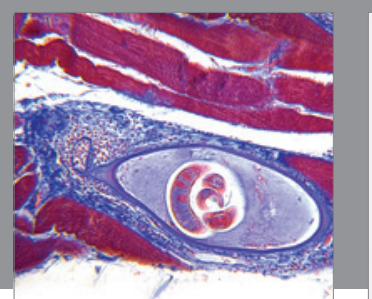

Gastroenterology Research and Practice

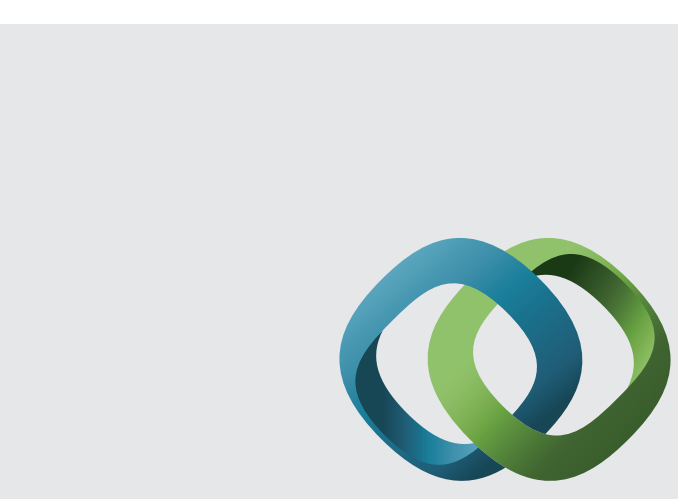

\section{Hindawi}

Submit your manuscripts at

http://www.hindawi.com
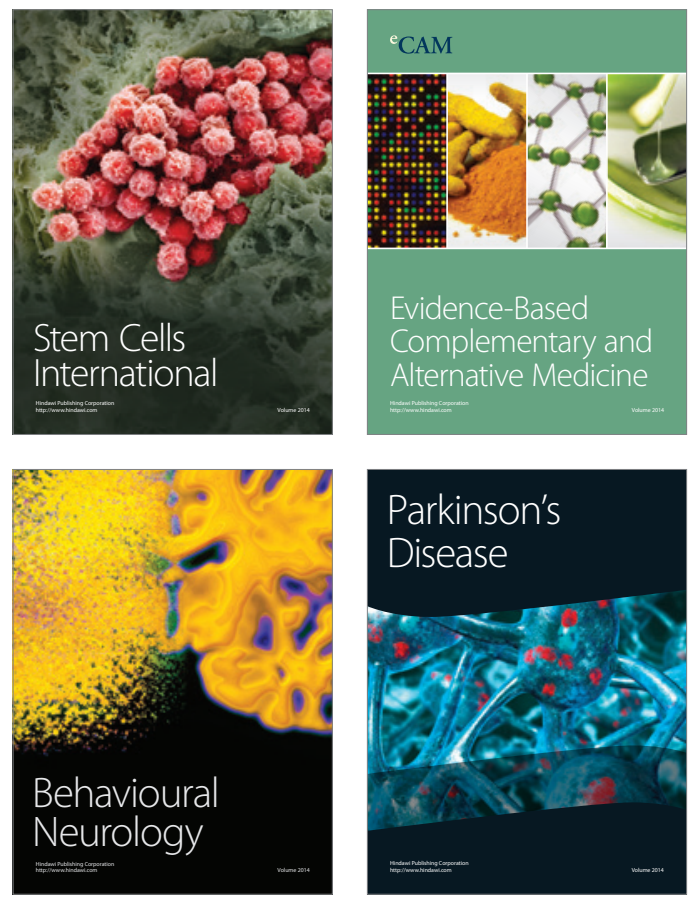
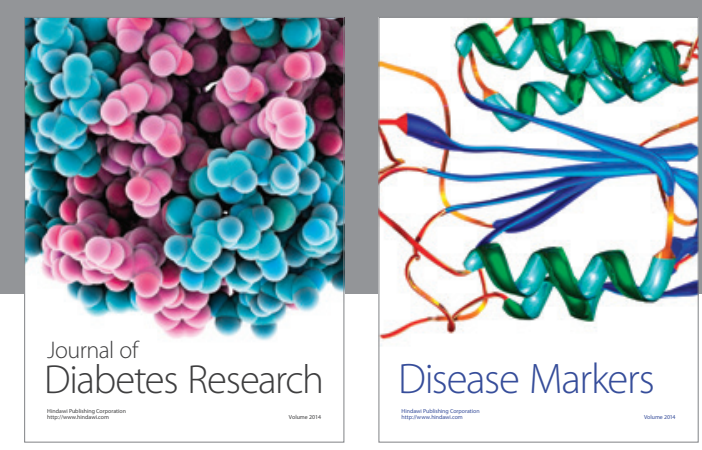

Disease Markers
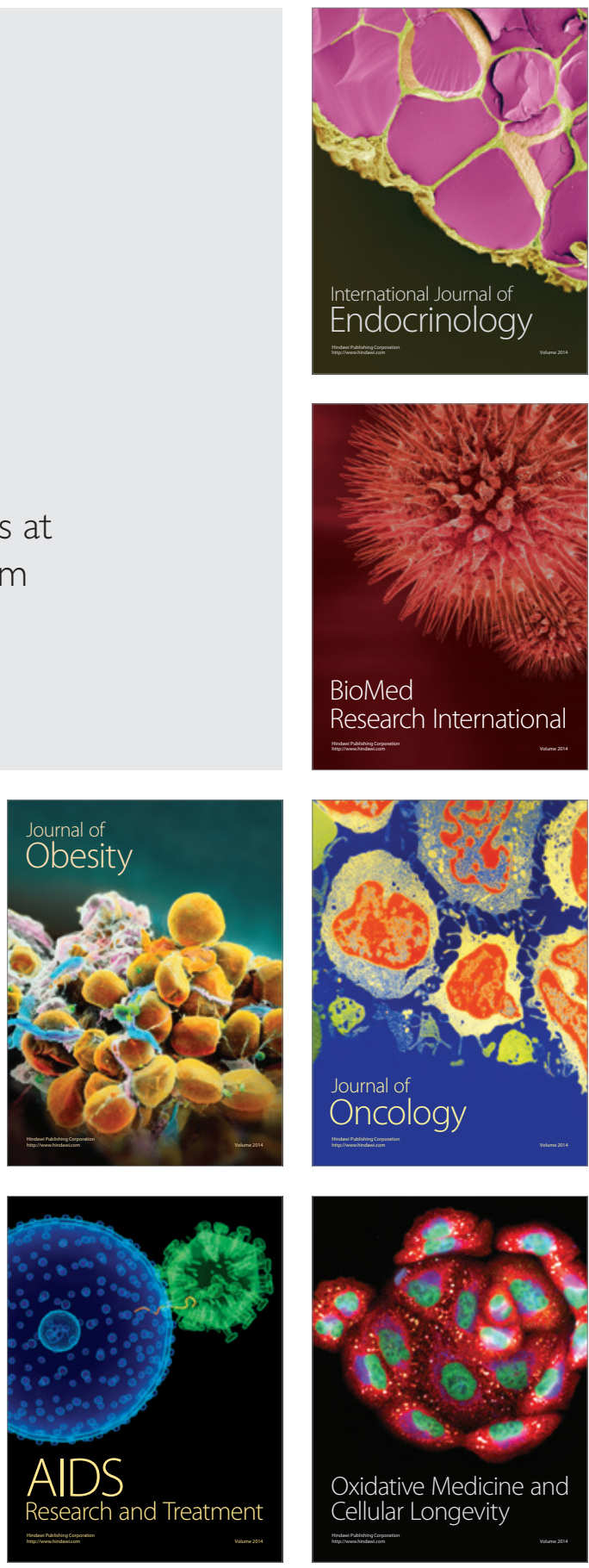